УДК 621.565

Хасан Весам Анвар Али

Одесская национальная академия пищевых технологий, ул. Канатная, 112, г. Одесса, 65039, Украина

\e-mail: dr_alshabi@hotmail.com

\title{
КРИОХИРУРГИЧЕСКИЕ АППАРАТЫ, РАБОТАЮЩИЕ НА ЭТИЛОВОМ СПИРТЕ
}

В статье рассмотрены герметичные криохирургические аппараты, работаюшие на твердом этиловом спирте. Приведены уравнения, определяющие тепловые потоки, проходящие от замораживаемого объекта через криозонд (медный стержень) к жидкому и, далее, к твердому спирту. Для описания проиесса охлаждения, замораживания и переохлаждения твердого объекта криохирургии введено понятие эквивалентной теплопроводности. Определены изменяющиеся во времени тепловые потоки, толщина жидкого спирта и температурные потери по криозонду и жидкому спирту. Приведенные расчетные уравнения позволяют выбрать конструкцию аппарата для различных объектов криохирургии.

Ключевые слова: Криохирургический аппарат; Криозонд; Рабочее тело; Этиловый спирт; Теплопроводность.

Хасан Весам Анвар Алі

Одеська національна академія харчових технологій, вул. Канатна, 112, м. Одеса, 65039, Україна

\e-mail: dr_alshabi@hotmail.com

\section{КРІОХІРУРГИЧНІ АПАРАТИ, ЯКІ ПРАЦЮЮТЬ НА ЕТИЛОВОМУ СПИРТІ}

В статті розглядаються герметичні кріохірургічні апарати з твердим етиловим спиртом. Приведені рівняння, які визначають теплові потоки, що проходять від об 'єкта, що заморожується, через кріозонд (мідний стрижень) до зрідженого $i$, далі, до твердого спирту. Для опису процесів охолоджування, заморожування та переохолоджування твердого об'єкта кріохірургії введено поняття еквівалентної теплопровідності. Виявлено теплові потоки, які змінюються у часі, товщина зрідженого спирту та температурні втрати по кріозонду та зрідженому спирту. Приведені рівняння дозволяють обрати конструкиію апарата для різних об'єктів кріохірургії.

Ключові слова: Кріохірургічні апарати; Кріозонд; Робоче тіло; Етиловий спирт; ТеплопровідHicmb.

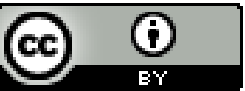

\section{I. ВВЕДЕНИЕ}

Криохирургические аппараты находят широкое применение в медицине. Наиболее распространенными являются открытые аппараты, в которых в качестве рабочего тела применяется жидкий азот с фазовым переходом жидкость - пар. Особенность открытых аппаратов заключается в том, что образующий при работе аппарата пар отводится в окружающую среду и жидкий азот необходимо пополнять после проведения криооперации. Это основной недостаток таких аппаратов.

Сравнительно недавно был предложен аппарат, в котором для получения холода используется рабочее тело с фазовым переходом жидкость-твердое тело [1] Так как объемы жидкости и твердого тела отличаются незначительно, то в таких аппаратах контейнер, где хранится рабочее тело, может быть изготовлен герметичным. Такой аппарат относится к классу закрытых аппаратов и рабочее тело в нем не расходуется. Однако, в этих аппаратах перед проведением криооперации рабочее тело должно охлаждаться и замораживаться. Для этого нужны простые криогенные машины, работающие на смесях холодильных агентов [2].

\section{II. ТЕОРЕТИЧЕСКИЙ АНАЛИЗ АППАРАТОВ}

На Рисунке 1 и Рисунке 2 показаны конструкции таких аппаратов с неоребренным и оребренным медным стержнем - криозондом, связывающим контейнер, в котором хранится рабочее тело, с объектом криооперации.

В качестве рабочего тела, заливаемого в герметичный контейнер, можно принять этиловый спирт. Теплофизические свойства этилового спирта приведены в Таблице 1, [3].

Таблица 1 - Теплофизические свойства этилового спирта.

\begin{tabular}{|c|c|c|c|c|c|}
\hline 突 & 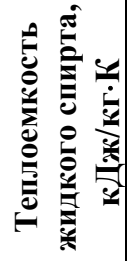 & 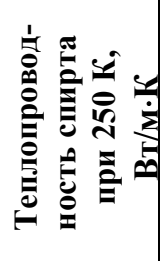 & 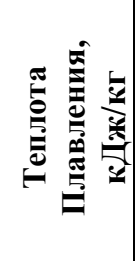 & 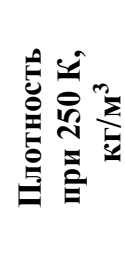 & 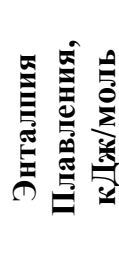 \\
\hline 56 & 2.332 & 0.187 & 108 & 784 & 4.81 \\
\hline
\end{tabular}




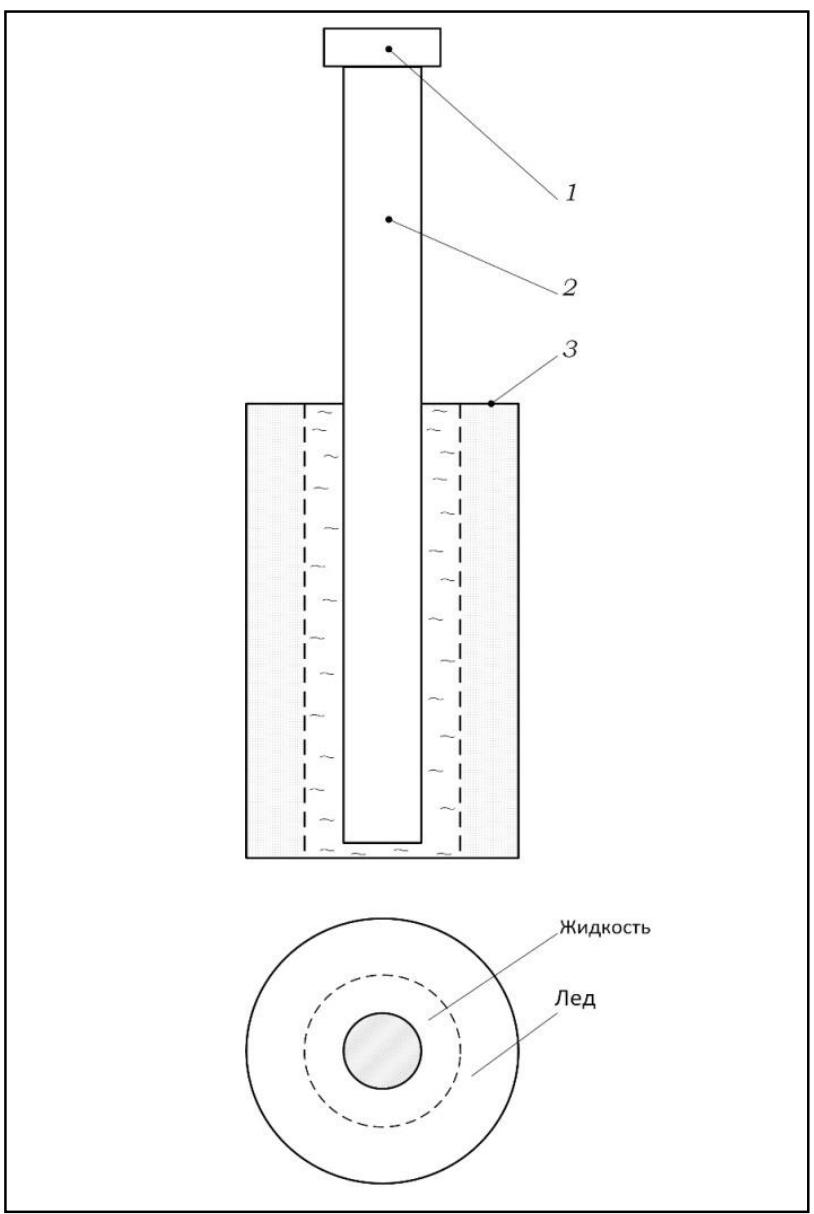

Рисунок 1 - Схема аппарата с неоребренным криозондом:

1 -наконечник, 2 -медный стержень, 3 -контейнер

Приведем расчетные уравнения аппарата. Теплофизические свойства человеческой биологической ткани близки к свойствам упитанной свинины, свойства которой приведены в Таблице 2, [4].

Таблица 2 - Теплофизические свойства биологической ткани свининь.

\begin{tabular}{|c|c|c|c|c|}
\hline 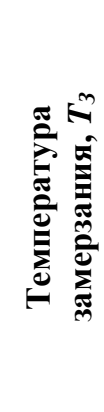 & 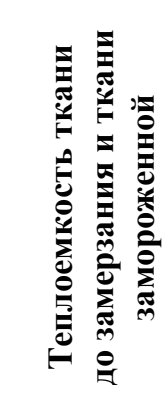 & 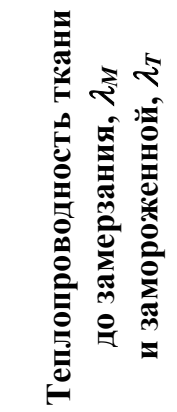 & 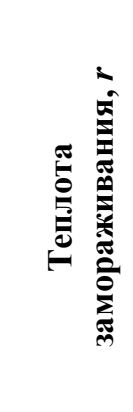 & $\begin{array}{l}0 \\
\hat{0} \\
0 \\
0 \\
0 \\
0\end{array}$ \\
\hline К & кДж/кг·К & Вт/м. K & кДж/кг & $\kappa \Gamma / \mathbf{M}^{3}$ \\
\hline 270.8 & $2.85-1.59$ & $\begin{array}{l}0.72--1.56 \\
0.32--0.49\end{array}$ & 154 & 950 \\
\hline
\end{tabular}

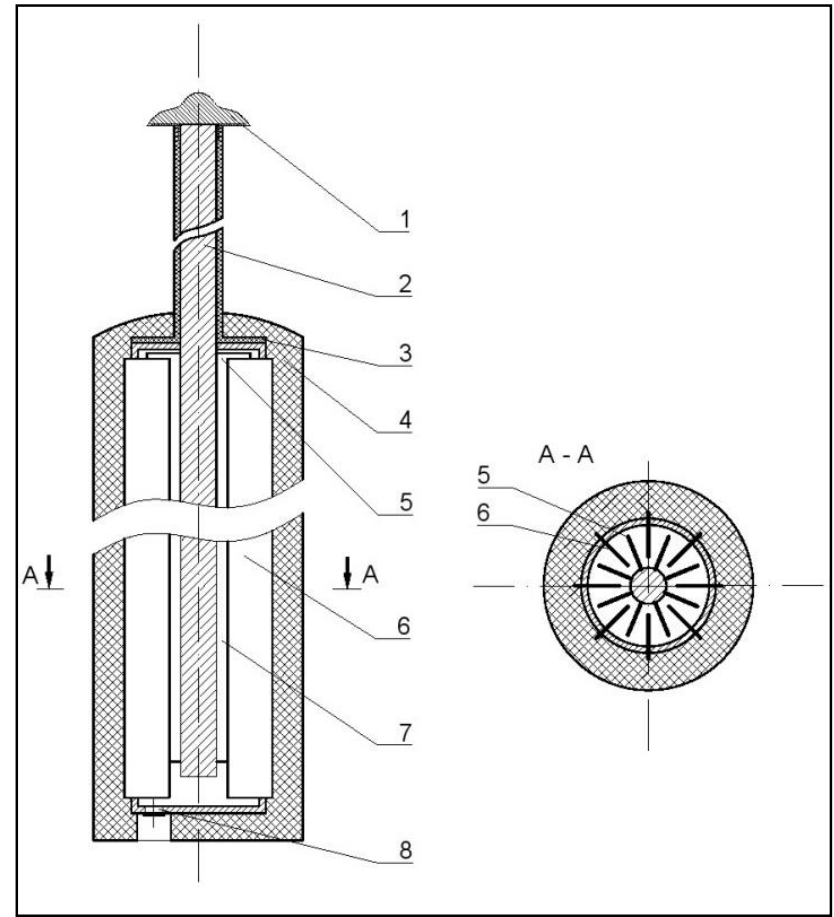

Рисунок 2 - Схема аппарата с оребренным криозондом

1 - наконечник, 2 -медный криозонд, 3 -контейнер, 4 - теплоизоляция, 5 и 7-ребра криозонда, 6 - ребра контейнера, 8-заливное отверстие.

Сначала определим, какое количество тепла $Q$ (кДж/кг) нужно отвести при охлаждении, замораживании и переохлаждении ткани $-Q_{1}, Q_{2}$ и $Q_{3}$.

$$
Q=Q_{1}+Q_{2}+Q_{3} \text {, }
$$

Здесь

$$
\left\{\begin{array}{l}
Q_{1}=m \cdot C\left(T_{C}-T_{3}\right), \\
Q_{2}=m \cdot r, \\
Q_{3}=m \cdot C_{T}\left(T_{3}-T_{K}\right)
\end{array}\right.
$$

В системе уравнений (2) обозначено: $m$ - масса обрабатываемого криообъекта, $C$ и $C_{T}-$ удельные теплоемкости охлаждаемой и затвердевшей ткани, $r$ - теплота замораживания ткани, $T_{C}-$ начальная температура криообъекта, $T_{3}$ - температура замерзания объекта и $T_{K}$ - заданная конечная температура замораживания объекта.

Кроме количества тепла необходимо знать и тепловую мощность. Для этого допустим, что время проведения криооперации составляет некоторую заданную величину $\tau$, Тогда тепловая мощность (холодопроизводительность) $Q_{0}$, необходимая для замораживания объекта за заданное время $\tau$, составит:

$$
Q_{0}=\frac{Q}{\tau}, \text { Вт }
$$

Эта мощность, отводимая от криообъекта, неравномерна во времени, проходит по криозонду и плавит лед этилового спирта. Потоки тепла от охлаждения, замораживания и переохлаждения твердой 
ткани разделены во времени. Реально все эти процессы проходят одновременно и точно рассчитать их очень трудно. Для упрощения этой задачи введем понятие эквивалентной теплопроводности $\lambda_{\text {э}}$, которую определим из следующего уравнения:

$$
\frac{Q_{1}+Q_{2}+Q_{3}}{\tau}=f_{C} \frac{\lambda_{\ni}}{l_{C}}\left(T_{C}-T_{K}\right),
$$

где $f_{C}$ и $l_{C}-$ площадь замораживаемой ткани и ее толщина.

$$
\begin{aligned}
& \text { Из уравнений (2), (3) и (4) следует, что } \\
& \lambda_{\text {э }}=\frac{m\left[C\left(T_{C}-T_{3}\right)+r+C_{T}\left(T_{3}-T_{K}\right)\right] l_{C}}{\tau \cdot f_{C}\left(T_{C}-T_{K}\right)}
\end{aligned}
$$

Согласно схеме аппарата (без оребрения стержня), запишем систему уравнений, описывающих процесс теплопередачи от криообъекта ко льду этилового спирта:

$$
\left\{\begin{array}{l}
Q_{0}=f_{C} \frac{\lambda_{\ni}}{l_{C}}\left(T_{C}-T_{H}\right), \\
Q_{0}=f_{C T} \frac{\lambda_{C T}}{l_{C T}}\left(T_{H}-T_{1}\right), \\
Q_{0}=f_{Ж} \frac{\lambda_{Ж}}{l_{Ж}}\left(T_{1}-T_{\text {Пл }}\right)
\end{array}\right.
$$

Здесь $f_{C T}$ - площадь сечения криозонда, находящегося вне контейнера и имеющего диаметр $d_{C T}$ $\left(f_{C T}=\pi \cdot d_{C T}^{2} / 4\right) ; l_{C T}-$ длина криозонда между криообъектом (наконечникоми) и контейнером со льдом этилового спирта; $T_{1}$ - температура криозонда внутри контейнера; $T_{П л}-$ температура плавления льда; $T_{H}-$ температура наконечника криозонда, которая при подготовке к операции и во время операции при контакте с объектом проходит вначале от температуры 156 K до 309,6 К, а затем падает до температуры замерзания $T_{3}$ и далее до заданной температуры замораживания объекта криохирургии $(253 \mathrm{~K}) ; l_{ж}-$ толщина слоя жидкого спирта; $\lambda_{ж}-$ теплопроводность жидкого спирта, $f_{ж}-$ поверхность медного стержнязонда внутри контейнера, которая обменивается теплом с жидким спиртом:

$$
f_{\mathscr{H}}=\pi \cdot d_{C T} \cdot L,
$$

здесь $L$ - длина стержня внутри контейнера.

Запишем систему уравнений (6) в следующем виде:

$$
\left\{\begin{array}{l}
T_{C}-T_{H}=\frac{Q_{0} \cdot l_{C}}{f_{C} \cdot \lambda_{\ni}}, \\
T_{H}-T_{1}=\frac{Q_{0} \cdot l_{C T}}{f_{C T} \cdot \lambda_{C T}}, \\
T_{1}-T_{\text {Пл }}=\frac{Q_{0} \cdot l_{Ж}}{f_{Ж} \cdot \lambda_{Ж}}
\end{array}\right.
$$

Суммируя уравнения системы (8), получим:

$$
T_{C}-T_{\text {Пл }}=Q_{0}\left(\begin{array}{l}
\frac{l_{C}}{f_{C} \cdot \lambda_{\ni}}+\frac{l_{C T}}{f_{C T} \cdot \lambda_{C T}} \\
+\frac{l_{Ж}}{f_{Ж} \cdot \lambda_{Ж}}
\end{array}\right)
$$

С другой стороны, можно записать выражения для определения количества жидкости расплавившегося спирта $M$ через тепловой поток $Q_{0}$ :

$$
M=\frac{Q_{0} \cdot \tau}{r} ; \text { или } M=V \cdot \rho,
$$

где $V$ - объем жидкого спирта; $r$ - теплота плавления твердого спирта.

$$
V=\pi \cdot d_{L} \cdot l_{\varkappa} \cdot L
$$

Здесь $d_{L}$ и $L$ - диаметр и длина криозонда внутри контейнера.

С учетом этих зависимостей имеем новую систему уравнений:

$$
\left\{\begin{array}{l}
T_{C}-T_{\text {Пл }}=Q_{0}\left(\frac{l_{C}}{f_{C} \cdot \lambda_{\ni}}+\frac{l_{C T}}{f_{C T} \cdot \lambda_{C T}}+\frac{l_{Ж}}{f_{Ж} \cdot \lambda_{Ж}}\right) \\
l_{\text {Ж }}=\frac{Q_{0} \cdot \tau}{r \cdot \rho \cdot \pi \cdot d_{L} \cdot L}
\end{array}\right.
$$

Решая систему уравнений (12), получим квадратное уравнение вида:

$$
a \cdot Q_{0}^{2}+b \cdot Q_{0}-c=0
$$

где $a$ и $b$ зависят от времени $\tau$, а $c$ - постоянная величина.

$$
\begin{gathered}
a=\frac{\tau}{r \cdot \rho \cdot \pi \cdot d_{L} \cdot L \cdot f_{\text {ж }} \cdot \lambda_{\varkappa}}, \\
b=\frac{l_{C}}{f_{C} \cdot \lambda_{\ni}}+\frac{l_{C T}}{f_{C T} \cdot \lambda_{C T}}, \\
c=T_{C}-T_{\text {ПЛ }} .
\end{gathered}
$$

Заметим, что толщина замораживаемого криообъекта $l_{C}$ и толщина жидкого этилового спирта $l_{ж}$ являются функциями времени.

Определим значения холодопроизводительности в начале работы аппарата $(\tau=0)$ и в конце работы (заданная величина $\tau=300$ сек).

В начале криооперации коэффициент $a=0$ и величина толщины промораживаемого криообъекта $l_{C}=$ 0 . Тогда при $\tau=0$ можно записать:

$$
b \cdot Q_{0}-c=0,
$$

где

$$
b=\frac{l_{C T}}{f_{C T} \cdot \lambda_{C T}}
$$


Известно, что дискриминант Д квадратного уравнения (13) равняется: Д $=b^{2}-4 a c$. Уравнение (13) имеет два следующих корня: $Q_{0}=\frac{-b \pm \sqrt{Д}}{2 a}$.

Действительным результатом будем считать положительный корень.

Таким образом, определены переменные тепловые потоки для заданной схемы теплопередачи через неоребренный криозонд.

Если стержень, расположенный в контейнере, оребрен, то основные расчетные соотношения остаются, в основном, такими же, как и при неоребренном стержне. Изменится только коэффициент $a$, который будет иметь вид:

$$
a=\frac{\tau}{r \cdot \rho \cdot\left(\pi \cdot d_{L}+2 \cdot n \cdot b^{\prime}\right) \cdot L \cdot f_{Ж} \cdot \lambda_{Ж}}
$$

здесь $n$ - число ребер и $b^{\prime}-$ ширина ребра (Рисунок 2).

\section{III. РАСЧЕТ АППАРАТА ДЛЯ КРИООПЕРАЦИИ МИНДАЛИНЫ}

Расчет проведем для случая неоребренного стержня.

Перед проведением операции снимаем с аппарата внешнюю теплоизоляцию и аппарат вместе со спиртом замораживаем в камере до температуры замерзания спирта (156 K).

Теплофизические свойства миндалины и спирта и конструктивные особенности аппарата приведены в Таблицах 1 и 2, а также на Рисунке 3 . По уравнению (2), задавшись массой миндалины $m=0,02$ кг и температурами $T_{C}=309,6 \mathrm{~K}, T_{3}=270,8 \mathrm{~K}, T_{H}=253 \mathrm{~K}$ и $T_{C \Pi}=156 \mathrm{~K}$, найдем, что $Q_{1}=2,21$ кДж, $Q_{2}=$ 3,08 кДж, $Q_{3}=0,566$ кДж и $Q=5,856$ кДж. При времени проведения криооперации $\tau$, равном 300 с, средняя холодопроизводительность равна $Q_{0}=19,52$ Вт. Через 300 с вся толщина миндалины, равная 0,005 м, заморожена.

Пренебрегаем термическими сопротивлениями между объектом криохирургии и наконечником криозонда (Тоб =Тн), а также между поверхностью криозонда внутри контейнера и жидким спиртом $\left(\mathrm{T}_{1}=\right.$ Тсп).

Допустим, что диаметр миндалины $d_{C}$ и диаметр верхней части криозонда (наконечника) $d_{C T}$ одинаковы и равны 0,012 м. Тогда, площадь заморозки объекта равна площади верхней части криозонда: $f_{C}=f_{C T}=$ $1,1310^{-4} \mathrm{~m}^{2}$. Коэффициент $c$ равен 153.6, а $\lambda_{\ni}=$ 15,256 Вт/м К. Теплопроводность меди известна 384 Вт/м К. Из Рис.3 видно: $l_{C T}=0,1$ м, диаметр стержня внутри контейнера $d_{L}=0,02$ м и длина стержня внутри контейнера $L=0,2$ м. Теплопроводность жидкого спирта равна 0,187 Вт/м К (Таблица 1).

Рассмотрим значения расчетных параметров при операции миндалины в любой момент времени в интервале $0 \ldots 300$ с. Для этого разобьём временной интервал на участки. Предположим, что толщина промороженной ткани изменяется с течением времени линейно от 0 до толщины миндалины $l_{C}=0,005$ м.

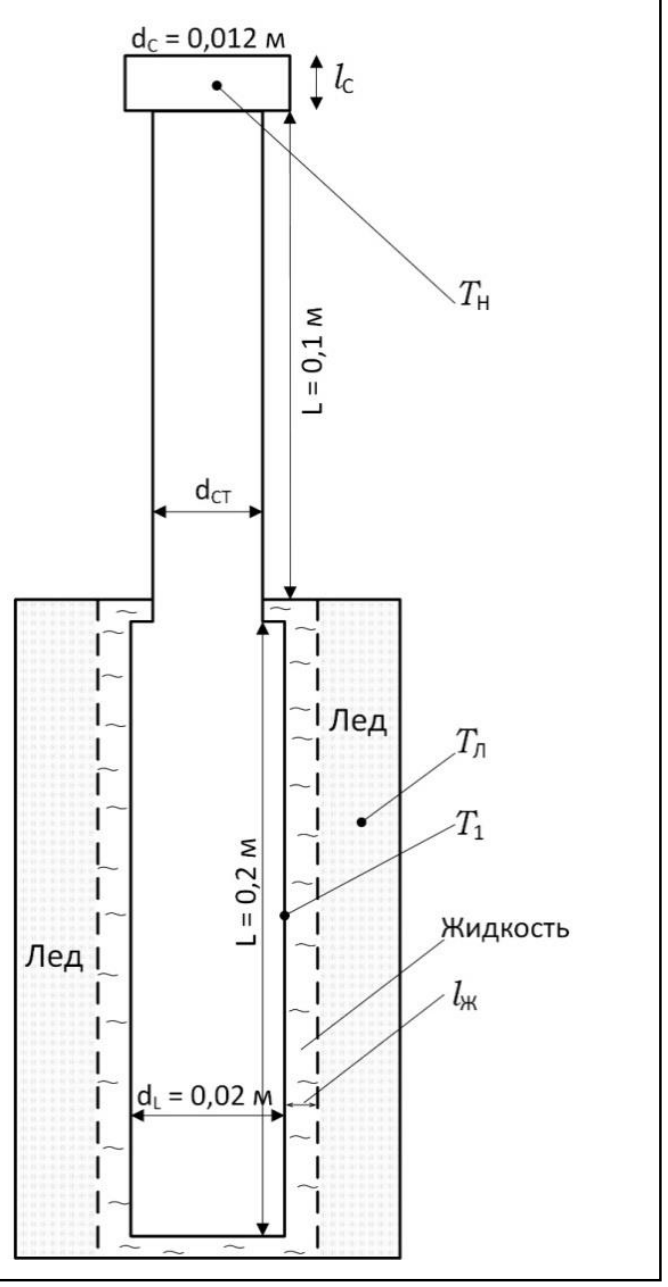

Рисунок 3 - Расчетная схема аппарата.

Результаты расчетов временной зависимости холодопроизводительности $Q_{0}$ и толщины слоя жидкости - $l_{ж}$, возникающей от плавления твердого спирта, приведены в Таблице 3 и на Рисунке 4.

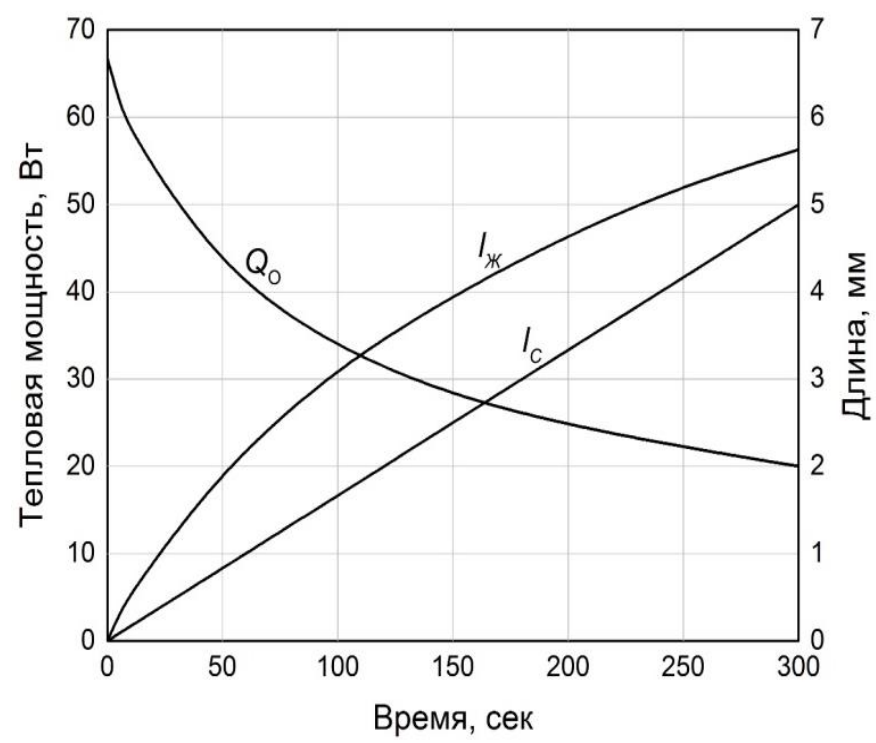

Рисунок 4-Зависимость $Q_{0}, l_{C}$ и $l_{ж}$ от времени. 
Таблица 3 - Значения расчетных параметров при операции миндалины в любой момент времени в интервале 0 ... 300 c.

\begin{tabular}{|c|c|c|c|c|c|}
\hline$\tau$ & $l_{C}$ & $a$ & $b$ & $Q_{0}$ & $l_{ж}$ \\
\hline сек & MM & -- & -- & BT & MM \\
\hline 0 & 0 & 0 & 2,3 & 66,78 & 0 \\
\hline 5 & 0,083 & 0,002 & 2,352 & 62,00 & 0,290 \\
\hline 10 & 0,166 & 0,004 & 2,400 & 58,25 & 0,547 \\
\hline 50 & 0,833 & 0,020 & 2,790 & 42,25 & 1,980 \\
\hline 100 & 1,666 & 0,040 & 3,270 & 33,45 & 3,140 \\
\hline 150 & 2,500 & 0,060 & 3,378 & 28,08 & 3,960 \\
\hline 200 & 3,332 & 0,080 & 4,230 & 24,73 & 4,650 \\
\hline 250 & 4,165 & 0,100 & 4,700 & 22,20 & 5,220 \\
\hline 300 & 5,000 & 0,120 & 5,260 & 20,00 & 5,630 \\
\hline
\end{tabular}

Определим температурные потери по толщине жидкого спирта ( $T_{1}$-Тпл) и по медному стержню между наконечником и контейнером, в котором хранитсяи спирт (температура $T_{H}$ ). Для этого систему уравнений (8) перепишем относительно температур:

$$
T_{1}=156+\frac{Q_{0} \cdot l_{Ж}}{f_{Ж} \cdot \lambda_{Ж}}
$$

$$
T_{H}=T_{1}+\frac{Q_{0} \cdot l_{C T}}{f_{C T} \cdot \lambda_{C T}}=T_{1}+2,3 \cdot Q_{0}
$$

или

$$
T_{H}=309,6-\frac{Q_{0} \cdot l_{C}}{f_{C} \cdot \lambda_{\ni}}
$$

Для проверки температуры $T_{H}$ рассчитаем ее по уравнениям (20) и (21).

Результаты расчетов температур приведены в Таблице 4.

Рассмотрение данных, приведенных в Таблице 3 и Таблице 4, показывает, что принятые допущения при выводе расчетных уравнений в незначительной мере искажают процессы, происходящие в аппарате, и приемлемы.

Холодопроизводительность аппарата уменьшается по мере промораживания объекта и по мере роста жидкой пленки спирта. Температура $T_{H}$, рассчитанная «сверху», т.е. с учетом термического сопротивления объекта (уравнение 21), удовлетворительно совпадает с температурой $T_{H}$, рассчитанной «снизу», т.е. с учетом термического сопротивления жидкого спирта и медного стержня между наконечником и контейнером

\begin{tabular}{|c|c|c|c|c|c|c|c|c|c|c|}
\hline$\tau$ & $Q_{0}$ & $l_{C}$ & $l_{\mathcal{K}}$ & $\frac{Q_{0} \cdot l_{Ж}}{f_{\mathscr{K}} \cdot \lambda_{\mathcal{W}}}$ & $T_{1}$ & $\frac{l_{C}}{f_{C} \cdot \lambda_{\ni}}$ & $\frac{Q_{0} \cdot l_{C}}{f_{C} \cdot \lambda_{\ni}}$ & $\begin{array}{c}T_{H} \\
y p-\text {-нuе } \\
(21)\end{array}$ & $2,3 \cdot Q_{0}$ & $\begin{array}{c}T_{H} \\
y p-\text {-ние } \\
(20)\end{array}$ \\
\hline сек & $\mathbf{B T}_{\mathbf{T}}$ & MM & MM & $\mathbf{K}$ & $\mathbf{K}$ & $\mathbf{K} / \mathbf{B} \mathbf{T}$ & $\mathbf{K}$ & $\mathbf{K}$ & $\mathbf{K}$ & $\mathbf{K}$ \\
\hline 0 & 66,78 & 0 & 0 & 0 & 156,0 & 0 & 0 & 309,6 & 153,0 & 309,6 \\
\hline 5 & 62,00 & 0,083 & 0,290 & 7,66 & 164,0 & 0,048 & 2,976 & 306,6 & 142,6 & 306,6 \\
\hline 10 & 58,25 & 0,166 & 0,547 & 13,60 & 169,6 & 0,097 & 5,627 & 303,9 & 134,0 & 303,6 \\
\hline 50 & 42,25 & 0,833 & 1,980 & 35,56 & 191,6 & 0,483 & 20,400 & 289,2 & 97,2 & 288,8 \\
\hline 100 & 33,45 & 1,666 & 3,140 & 44,70 & 201,0 & 0,966 & 32,300 & 277,3 & 76,9 & 277,9 \\
\hline 150 & 28,08 & 2,500 & 3,960 & 47,25 & 203,0 & 1,450 & 40,700 & 268,9 & 64,6 & 267,6 \\
\hline 200 & 24,73 & 3,332 & 4,650 & 48,96 & 205,0 & 1,930 & 47,700 & 261,9 & 56,9 & 262,0 \\
\hline 250 & 22,20 & 4,165 & 5,220 & 49,38 & 205,5 & 2,410 & 53,500 & 256,1 & 51,0 & 256,5 \\
\hline 300 & 20,00 & 5,000 & 5,630 & 48,10 & 204,0 & 2,900 & 58,000 & 251,6 & 46,0 & 250,0 \\
\hline
\end{tabular}
(уравнения 20 и 19) (Рисунок 5).

Таблица 4 - Результаты расчетов температур.

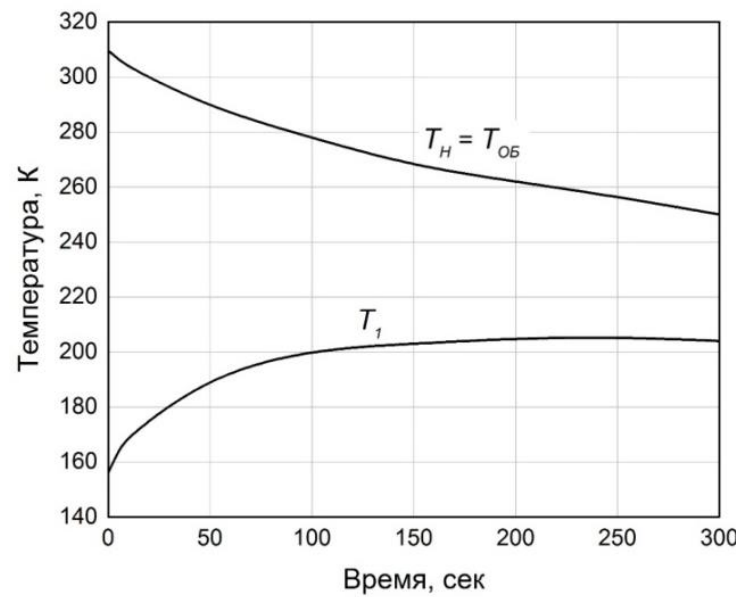

Рисунок 5 - Зависимость температур криозонда и объекта от времени

\section{ЗАКЛЮЧЕНИЕ}

Преимущество рассматриваемых аппаратов, работающих на твердом этиловом спирте, заключается в герметичной конструкции контейнера, которая дает возможность многократного их использования без замены рабочего тела.

Введение понятия эквивалентной теплопроводности дает возможность приближенно описать сложные процессы охлаждения, заморозки и переохлаждения твердого объекта криохирургии, а также тепловые процессы в криозонде и в контейнере, в котором хранится рабочее тело.

Приведенные уравнения позволяют провести тепловой расчет криоаппарата и выбрать конструкцию аппарата для различных объектов криохирургии. 


\section{ЛИТЕРАТУРА}

1. В.А. Наер, А.В. Роженцев, Хасан Весам Анвар Али, Криохирургические аппараты - аккумуляторы холода. - Холодильная техника и технология, 2014, №6(152), c.14 -19.

2. В.А. Наер, А.В. Роженцев, Хасан Весам Анвар Али, “Джоуль-томсоновские рефрижераторы с многокомпонентными рабочими телами”, Технические газы, 2014, №2, с. 11-19.
3. В.А. Наер, А.В. Роженцев, Хасан Весам, Анвар Али. Рабочие вещества с фазовым переходом жидкость-твердое тело-жидкость для криохирургических аппаратов. - Холодильная техника и технология, 2013, №6, c. 16-23.

4. А.В. Бараненко, В.С. Калюнов Б.Н. Малеванный, А.Я. Эглит. Практикум по холодильному технологическому оборудованию. Учебное пособие. СПб ГУН и ПТ, 2002, 170 с.

Отримана в редакції 15.01.2016, прийнята до друку 03.03.2016

\section{Hasan Vesam Anvar Ali $\bowtie$}

Odessa National Academy of Food Technologies, 112 Kanatnaia str., Odessa, 65039, Ukraine

$\triangle$ e-mail: dr_alshabi@hotmail.com

\section{CRYOSURGICAL APPARATUS OPERATING WITH ETHANOL}

The article deals with sealed cryosurgical devices operating on the solid ethyl alcohol. The equations that define the heat flows passing from the object which is freezing through the cryocatheter (copper rod) to the liquid and then to a solid alcohol are given. To describe the process of cooling, freezing and undercooling of solid object the concept of equivalent thermal conductivity was introduced in cryosurgery. Varying in time heat fluxes, the thickness of the liquid alcohol and temperature losses on cryocatheter and liquid alcohol were defined. These estimated equations allow you to select the device design for various cryosurgery objects, e.g. apparatus for cryosurgical operation of tonsillar. The calculations were performed for the case of non-finned rod. Before the operation the unit was removed from the outer insulation and the apparatus together with the alcohol in the chamber was frozen to the alcohol freezing point $(156 \mathrm{~K})$.

Keywords: Cryosurgical apparatus; Cryocatheter; Working fluid; Ethyl alcohol; Thermoconductivity.

\section{REFERENCES}

1. Naer, V.A., Rozhencev, A.V., Hasan Vesam Anvar Ali. (2014). Cryosurgical apparatus - accumulators of cold. Refrigeration engineering and technology, No. 6(152), 14-19.

2. Naer, V.A., Rozhencev, A.V., Hasan Vesam Anvar Ali (2014). Dzhoul'-tomsonovskie refrizheratory s mnogokomponentnymi rabochimi telami. Tekhnicheskie gazy, No.2, 11-19.
3. Naer, V.A., Rozhencev, A.V., Hasan Vesam, Anvar Ali. (2013). Working agents with phase transition liquidsolid-liquid for cryosurgical apparatus. Refrigeration engineering and technology, No. 6, 16-23.

4. Baranenko, A.V., Kalyunov, V.S., Malevannyj, B.N., Ehglit, A.Ya. (2002). Praktikum po holodil'nomu tekhnologicheskomu oborudovaniyu. Uchebnoe posobie. SPb GUN i PT, $170 \mathrm{p}$. 